\title{
Clinicopathological relevance of tumor expression of NK group 2 member D ligands in resected non-small cell lung cancer
}

\author{
Riki Okita', Ai Maeda', Katsuhiko Shimizu' ${ }^{1}$, Yuji Nojima', Shinsuke Saisho ${ }^{1}$ and \\ Masao Nakata' \\ ${ }^{1}$ Department of General Thoracic Surgery, Kawasaki Medical School, Kurashiki, Japan \\ Correspondence to: Riki Okita, email: riki0716okita@yahoo.co.jp \\ Keywords: non-small cell lung cancer (NSCLC); UL 16-binding protein (ULBP); prognostic factor; MICA/B (MHC class I chain-related \\ molecule A and B); NK cell \\ Received: June 17, $2019 \quad$ Accepted: September 24, $2019 \quad$ Published: November 26, 2019 \\ Copyright: Okita et al. This is an open-access article distributed under the terms of the Creative Commons Attribution License 3.0 \\ (CC BY 3.0), which permits unrestricted use, distribution, and reproduction in any medium, provided the original author and source \\ are credited.
}

\section{ABSTRACT}

UL16-binding protein (ULBP) 1-6 and MHC class I chain-related molecule A and B (MICA/B) are NK group 2, member D (NKG2D) ligands, which are specifically expressed in infected or transformed cells and are recognized by NK cells via NKG2DNKG2D ligand interactions. We previously reported that MICA/B overexpression predicted improved clinical outcomes in patients with resected non-small cell lung cancer (NSCLC). However, the clinicopathological features and prognostic significance of ULBPs in NSCLC remain unclear. Here,ULBP1-6 expression was evaluated based on immunohistochemistry of 91 NSCLC samples from patients following radical surgery. ULBPs were expressed by the majority of NSCLC. Either ULBP1 or ULBP2/5/6 overexpression was associated with squamous-cell carcinoma histology, whereas ULBP4 overexpression was associated with younger age and adenocarcinoma histology. Although overexpression of ULBP1-6 did not impact clinical outcomes in NSCLC patients, integrative profiling with cluster analysis classified patients into 3 subgroups based on the expression pattern of NKG2D ligands. The subgroup characterized by ULBP1 or ULBP2/5/6 high expressing but ULBP4 low expressing tumors showed poor overall survival. Taken together with previous results, NSCLC histological subtype strongly correlates with NKG2D ligands expression pattern. NKG2D ligands expression levels assessed by multiple immune parameters could predict clinical outcomes of patients with NSCLC.

\section{INTRODUCTION}

Lung cancer is the leading cause of cancer-related deaths worldwide [1]. Although TNM classification is the principal guide used for the prognostic evaluation of non-small cell lung cancer (NSCLC) [2], several immunological factors such as tumor infiltrating lymphocytes [3] or molecules related to immunerecognition such as human leukocyte antigen-A2 [4] are also suitable in order to predict the clinical outcome of patients with NSCLC. The use of immune checkpoint inhibitors is considered to be a highly effective therapeutic strategy for patients with NSCLC $[5,6]$, which prompted interest in evaluating the expression of $\mathrm{T}$ cell-related immunological factors such as Programmed cell death-1 (PD-1) ligand 1 (PD-L1) both in tumor cells and immune cells [7]. On the other hand, the role of NK cell-related immunological factors in NSCLC remains unclear, although NK cell count is a predictive factor for clinical benefit of PD-1 targeted therapy in melanoma [8].

The main role of $\mathrm{NK}$ cells is considered to be immunosurveillance [9]. NK group 2, member $\mathrm{D}$ (NKG2D) ligands consist of MHC class I chainrelated molecule $\mathrm{A}$ and $\mathrm{B}(\mathrm{MICA} / \mathrm{B})$ and UL16-binding protein (ULBP) 1-6, which promote NK cell-mediated cytotoxicity via the NK cell activating receptor NKG2D 
[10] and are expressed in transformed or infected cells [11]. Hypothetically, NK cell-mediated cytotoxicity against tumor cells should be enhanced if the NKG2D ligand is overexpressed in tumor cells. Indeed, NKG2D ligand overexpression was reported to be correlated with a better prognosis in several types of cancer [12-15]. In NSCLC, we previously reported that overexpression of MICA/B predicted improved clinical outcomes for resected NSCLC patients [16]. However, there is no report evaluating the correlation between ULBP1-6 expression and clinical outcome in patients with NSCLC, although high concentrations of serum-soluble ULBP2 in NSCLC patients were reported to be correlated with poor prognosis [17].

In this study, we evaluated the expression of ULBP1-6 using immunohistochemistry for samples from patients with resected, pathological stage (pStage) IA-IIIA NSCLC, using the same dataset as for our previous "MICA/B" study [16], and assessed the relationship between the expression status of each ULBP and patient characteristics or clinical outcomes. Additionally, previously described data from the "MICA/B" study [16] was updated in order to compare the clinical impact of MICA/B expression status with that of ULBPs. Our results showed that ULBP1 and ULBP2/5/6 are predominantly expressed in lung squamous cell carcinoma, while ULBP4 is expressed in lung adenocarcinoma. These findings suggest that ULBP1 and ULBP2/5/6 are promising targets for the treatment of lung squamous cell carcinoma while ULBP4 is one for the treatment of lung adenocarcinoma. Although overexpression of ULBPs has less impact on the survival of patients with resected NSCLC than overexpression of $\mathrm{MICA} / \mathrm{B}$, cluster analysis showed that the subgroup which was characterized by ULBP1 or ULBP2/5/6 high expressing but ULBP4 low expressing tumors showed poor overall survival.

\section{RESULTS}

\section{Relationship between ULBP expression and clinical characteristics of NSCLC}

Representative immunohistochemical stains for ULBPs are shown in Figure 1A. The expected cutoff values for the score of each molecule according to Receiver operating characteristic (ROC) curves (Supplementary Figure 1) are as follows: ULBP1: Score 1, ULBP2/5/6 Score 0: ULBP3: score 0, and ULBP4: score 2. From a total of 91 tumors, overexpression of ULBP1, ULBP2/5/6, ULBP3, and ULBP4 was found in $44(48.4 \%), 45(49.5 \%), 20(22.0 \%)$, and $63(69.2 \%)$ cases, respectively (Figure 1B), while that of MICA/B was found in $28(30.8 \%)$ cases, as previously described [16]. The clinicopathological characteristics of NSCLC samples are summarized in Supplementary Table 1. Interestingly, either ULBP1 or ULBP $2 / 5 / 6$ overexpression was correlated with a squamous cell carcinoma histology, while ULBP4 overexpression was correlated with younger age and adenocarcinoma histology (Table 1).

\section{Recurrence free survival (RFS) and overall survival (OS) stratified by ULBP expression status in resected NSCLC}

In order to investigate the correlation between ULBP expression and clinical outcomes, both RFS and OS were stratified using ULBP expression and evaluated. The results indicate that ULBP overexpression does not have an effect on RFS or OS (Figure 2A-D), while MICA/B overexpression was correlated with improved outcomes in both RFS and OS (Figure 2E). Although the original report showed that MICA/B overexpression could be used to predict improved outcomes in only RFS [16], if the follow-up period was updated to be the same length as that for ULBP, the effect on OS was found.

\section{RFS and OS stratified by multiple immune parameters in resected NSCLC}

In order to assess the impact of the overexpression of multiple NKG2D ligands on clinical outcome, RFS and OS were stratified by determining the number of overexpressed NKG2D ligands. Unexpectedly, the number of overexpressed NKG2D ligands had no impact on either RFS or OS (Figure 3). To assess the multiple immune parameters further, integrative profiling with cluster analysis classified our patients into 3 subgroups (category 1,2 , and 3) based on the expression pattern of NKG2D ligands, including $\mathrm{MICA} / \mathrm{B}$ (Figure 4). Interestingly, category 3 , the subgroup which was mainly characterized by ULBP 1 or ULBP $2 / 5 / 6$ high expressing but ULBP4 low expressing tumors, showed poor OS compared with category 1 or 2 , although there was no impact on RFS (Figure 5).

\section{Category 3 immune parameters were independent prognostic factors for poor outcome in resected NSCLC}

Cox regression analysis was performed to determine the predictive value of clinical variables for RFS and OS. Univariate analysis showed that lymphatic invasion, vascular invasion, and lymph node metastasis were potential predictors of RFS. Multivariate analysis showed only lymph node metastasis to be a prognostic factor for poor outcome (hazard ratio (HR) 4.779, $p=0.009$ ) for RFS (Table 2). On the other hand, univariate analysis showed that pleural invasion, lymphatic invasion, vascular invasion, lymph node metastasis, and the category were potential predictors of OS. Surprisingly, multivariate analysis showed the category to be an independent prognostic factor for poor clinical outcome (HR 0.329, 

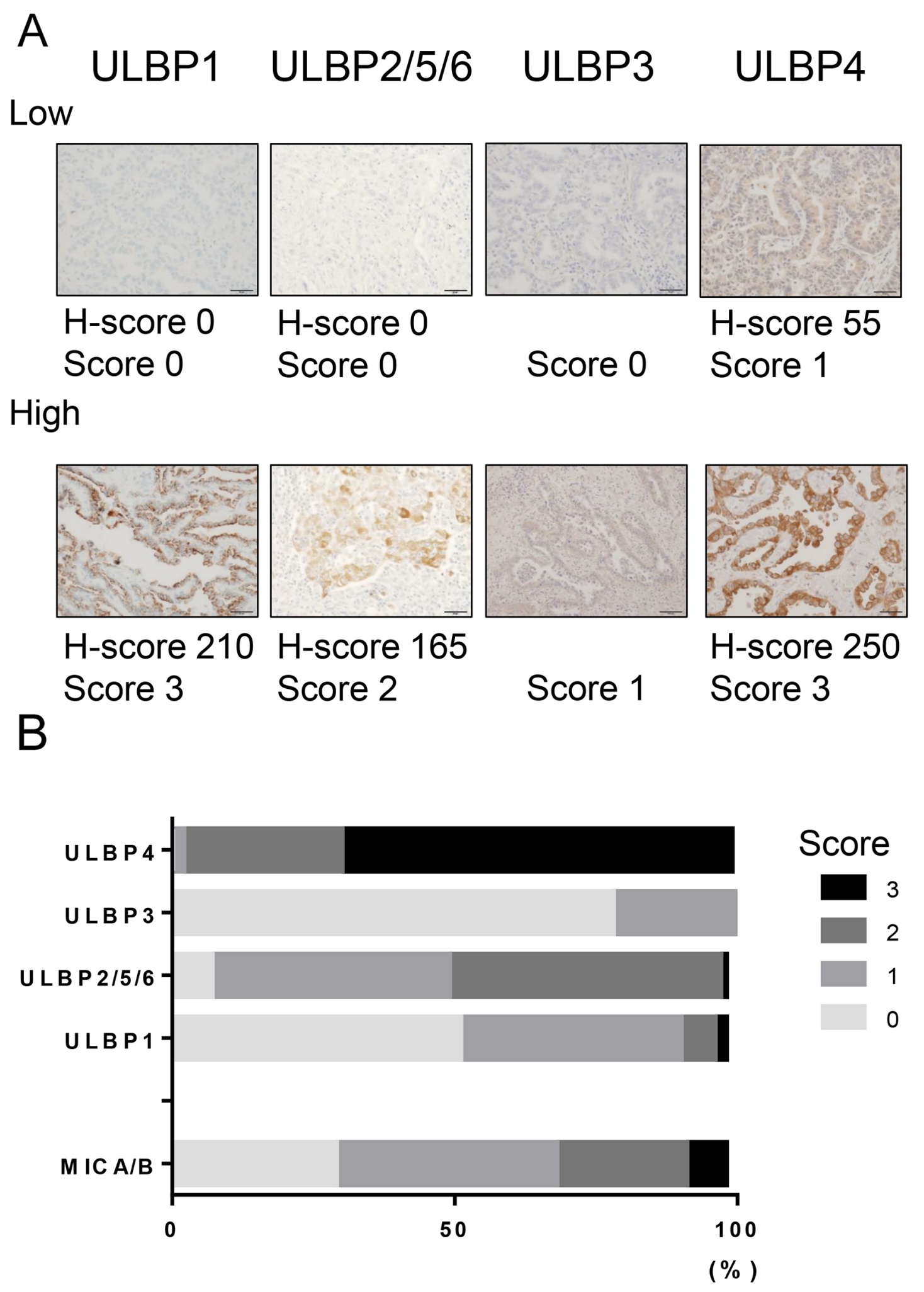

Figure 1: NKG2D ligand expression in NSCLC tissues. (A) Immunohistochemical staining of ULBPs in NSCLC tissues. Representative staining for ULBP1, ULBP2/5/6, ULBP3, and ULBP4 in tumor tissues (200× magnification). The panels show images corresponding to different intensity scores of NKG2D ligand expression. Low: score $0-1$ for ULBP1, $-2 / 5 / 6,-4$ or score 0 for ULBP3. High; score 2-3 for ULBP1, -2/5/6, -4 or score 1-3 for ULBP3. (B) Frequency of NKG2D ligand expression in non-small cell lung cancer. The graph shows the expression levels of each NKG2D ligand. 
Table 1: Clinicopathological characteristics by NKG2D ligand

\begin{tabular}{|c|c|c|c|c|c|c|c|c|c|c|c|c|c|c|}
\hline \multirow[t]{2}{*}{ Characteristic } & \multicolumn{2}{|c|}{ Number $(n=91)$} & \multicolumn{3}{|c|}{ ULBP1 } & \multicolumn{3}{|c|}{ ULBP2/5/6 } & \multicolumn{3}{|c|}{ ULBP3 } & \multicolumn{3}{|c|}{ ULBP4 } \\
\hline & & & low & high & $p$ & low & high & $p$ & low & high & $p$ & low & high & $p$ \\
\hline Age & $67.8(37-83)$ & & $66.6 \pm 8.6$ & $69.1 \pm 8.1$ & 0.165 & $68.8 \pm 7.2$ & $66.7 \pm 9.4$ & 0.241 & $67.7 \pm 8.2$ & $68.1 \pm 9.4$ & 0.872 & $71.3 \pm 7.1$ & $66.2 \pm 8.5$ & $0.007^{*}$ \\
\hline \multirow[t]{2}{*}{ Gender } & Male & $59(65 \%)$ & 27 & 32 & 0.127 & 26 & 33 & 0.093 & 48 & 11 & 0.297 & 20 & 39 & 0.380 \\
\hline & Female & $32(35 \%)$ & 20 & 12 & & 20 & 12 & & 23 & 9 & & 8 & 24 & \\
\hline \multirow[t]{2}{*}{ Smoking $^{\mathrm{a}}$} & Never & $33(37 \%)$ & 20 & 13 & 0.170 & 20 & 13 & 0.126 & 25 & 8 & 0.726 & 8 & 25 & 0.364 \\
\hline & $\begin{array}{l}\text { Current or } \\
\text { former }\end{array}$ & $57(63 \%)$ & 26 & 31 & & 25 & 32 & & 45 & 12 & & 19 & 38 & \\
\hline CEA (ng/ml) & & & $11.6 \pm 35.4$ & $6.4 \pm 11.3$ & 0.355 & $5.6 \pm 10.4$ & $12.6 \pm 36.3$ & 0.208 & $9.9 \pm 30.1$ & $4.2 \pm 4.0$ & 0.404 & $5.0 \pm 3.3$ & $10.8 \pm 31.9$ & 0.337 \\
\hline SUVmax & & & $8.2 \pm 6.1$ & $6.5 \pm 4.3$ & 0.126 & $7.7 \pm 5.7$ & $7.1 \pm 4.9$ & 0.604 & $7.1 \pm 5.5$ & $6.6 \pm 4.8$ & 0.708 & $6.8 \pm 5.8$ & $7.6 \pm 5.1$ & 0.469 \\
\hline $\begin{array}{l}\text { Tumor size } \\
(\mathrm{mm})\end{array}$ & & & $30.1 \pm 13.0$ & $30.7 \pm 13.5$ & 0.818 & $28.8 \pm 11.1$ & $32.0 \pm 15.0$ & 0.240 & $31.2 \pm 13.8$ & $27.5 \pm 10.7$ & 0.261 & $31.6 \pm 13.9$ & $29.9 \pm 12.9$ & 0.562 \\
\hline \multirow[t]{2}{*}{ Histology } & Adenocarcinom & $71(78 \%)$ & 41 & 30 & $0.028^{*}$ & 40 & 31 & $0.037^{*}$ & 54 & 17 & 0.394 & 18 & 53 & $0.035^{*}$ \\
\hline & $\begin{array}{l}\text { Squamous cell } \\
\text { carcinoma }\end{array}$ & $20(22 \%)$ & 6 & 14 & & 6 & 14 & & 17 & 3 & & 10 & 10 & \\
\hline \multirow[t]{3}{*}{$\begin{array}{l}\text { Histlogic } \\
\text { grade }\end{array}$} & G1 & $38(42 \%)$ & 20 & 18 & 0.611 & 24 & 14 & 0.126 & 30 & 8 & 0.264 & 9 & 29 & 0.327 \\
\hline & G2 & $29(32 \%)$ & 13 & 16 & & 12 & 17 & & 20 & 9 & & 9 & 20 & \\
\hline & G3 & $24(26 \%)$ & 14 & 10 & & 10 & 14 & & 21 & 3 & & 10 & 14 & \\
\hline \multirow[t]{2}{*}{$\begin{array}{l}\text { Pleural } \\
\text { invasion }\end{array}$} & Negative & $59(65 \%)$ & 30 & 29 & 0.836 & 33 & 26 & 0.163 & 46 & 13 & 0.986 & 19 & 40 & 0.687 \\
\hline & Positive & $32(35 \%)$ & 17 & 15 & & 13 & 19 & & 25 & 7 & & 9 & 23 & \\
\hline \multirow[t]{2}{*}{$\begin{array}{l}\text { Lymphatic } \\
\text { invasion }\end{array}$} & Negative & $66(73 \%)$ & 36 & 30 & 0.369 & 34 & 32 & 0.765 & 51 & 15 & 0.779 & 20 & 46 & 0.876 \\
\hline & Positive & $25(27 \%)$ & 11 & 14 & & 12 & 13 & & 20 & 5 & & 8 & 17 & \\
\hline \multirow[t]{2}{*}{$\begin{array}{l}\text { Vascular } \\
\text { invasion }\end{array}$} & Negative & $54(59 \%)$ & 27 & 27 & 0.704 & 30 & 24 & 0.249 & 43 & 11 & 0.655 & 13 & 41 & 0.095 \\
\hline & Positive & $37(41 \%)$ & 20 & 17 & & 16 & 21 & & 28 & 9 & & 15 & 22 & \\
\hline \multirow[t]{2}{*}{$\begin{array}{l}\text { Lymphnode } \\
\text { metastasis }\end{array}$} & N0 & $72(79 \%)$ & 38 & 34 & 0.675 & 38 & 34 & 0.408 & 58 & 14 & 0.256 & 23 & 49 & 0.636 \\
\hline & N1-2 & $19(21 \%)$ & 9 & 10 & & 8 & 11 & & 13 & 6 & & 5 & 14 & \\
\hline \multirow[t]{2}{*}{$\begin{array}{l}\text { Pathological } \\
\text { stage }\end{array}$} & IA & $35(38 \%)$ & 20 & 15 & 0.407 & 21 & 14 & 0.154 & 26 & 9 & 0.496 & 11 & 24 & 0.914 \\
\hline & IB-IIIA & $56(62 \%)$ & 27 & 29 & & 25 & 31 & & 45 & 11 & & 17 & 39 & \\
\hline
\end{tabular}

a:Data not available for one patient.

CEA: carcinoembryonic antigen, SUVmax: maximum standardized uptake value.

${ }^{*} p<0.05$

$p=0.009)$, while lymph node metastasis was not a significant prognostic factor (Table 3 ).

\section{DISCUSSION}

Recent developments in the use of immune checkpoint inhibitors as treatments have improved prognosis of advanced NSCLC [5, 6]. Nevertheless, 60$70 \%$ of patients receiving immune checkpoint therapy tend to develop tumor progression, which requires additional strategies for NSCLC treatment. NK cells play an important role in host immunity against the tumor, participating mainly in immune surveillance [9]. Recently, it was reported that NK cell count before PD-1/PD-L1 targeted therapy predicted treatment response in patients with melanoma [8]. Moreover, NK cell count decreased after regulatory $\mathrm{T}$ cell-depletion therapy using the antiCCR4 monoclonal antibody Mogamulizumab [20], suggesting that NK cells also affect immune checkpoint therapy. However, the clinical significance of NK cells 

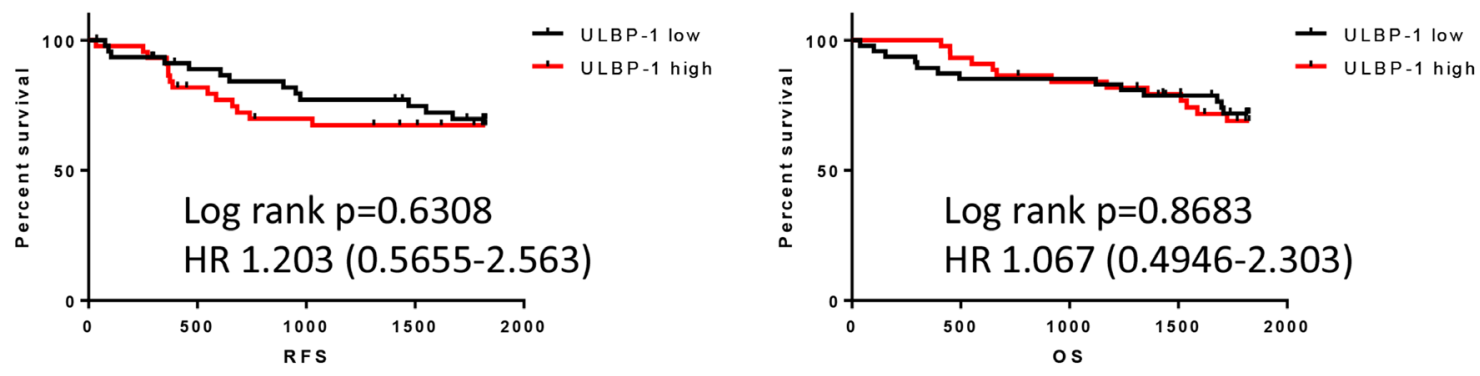

B
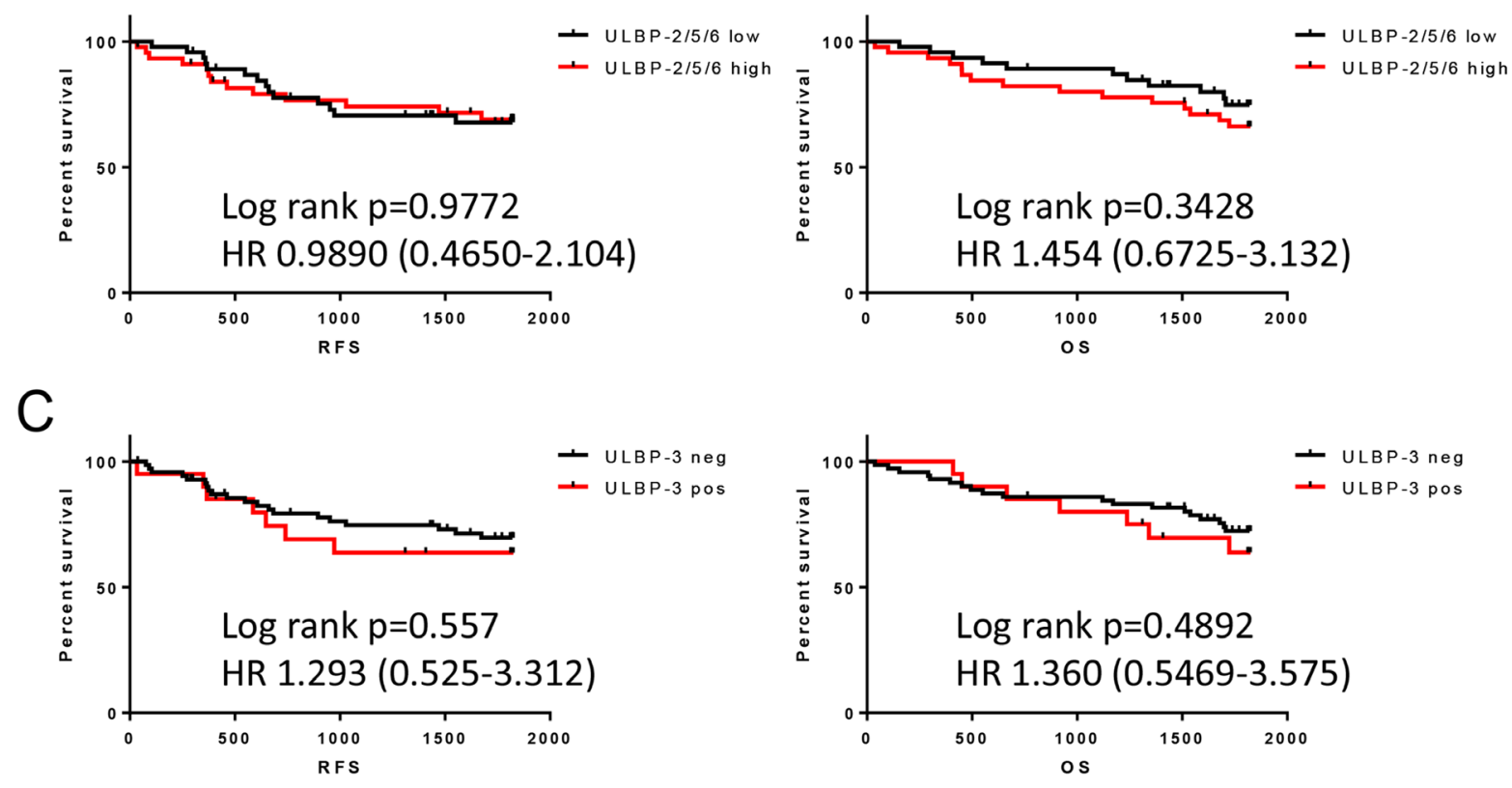

D
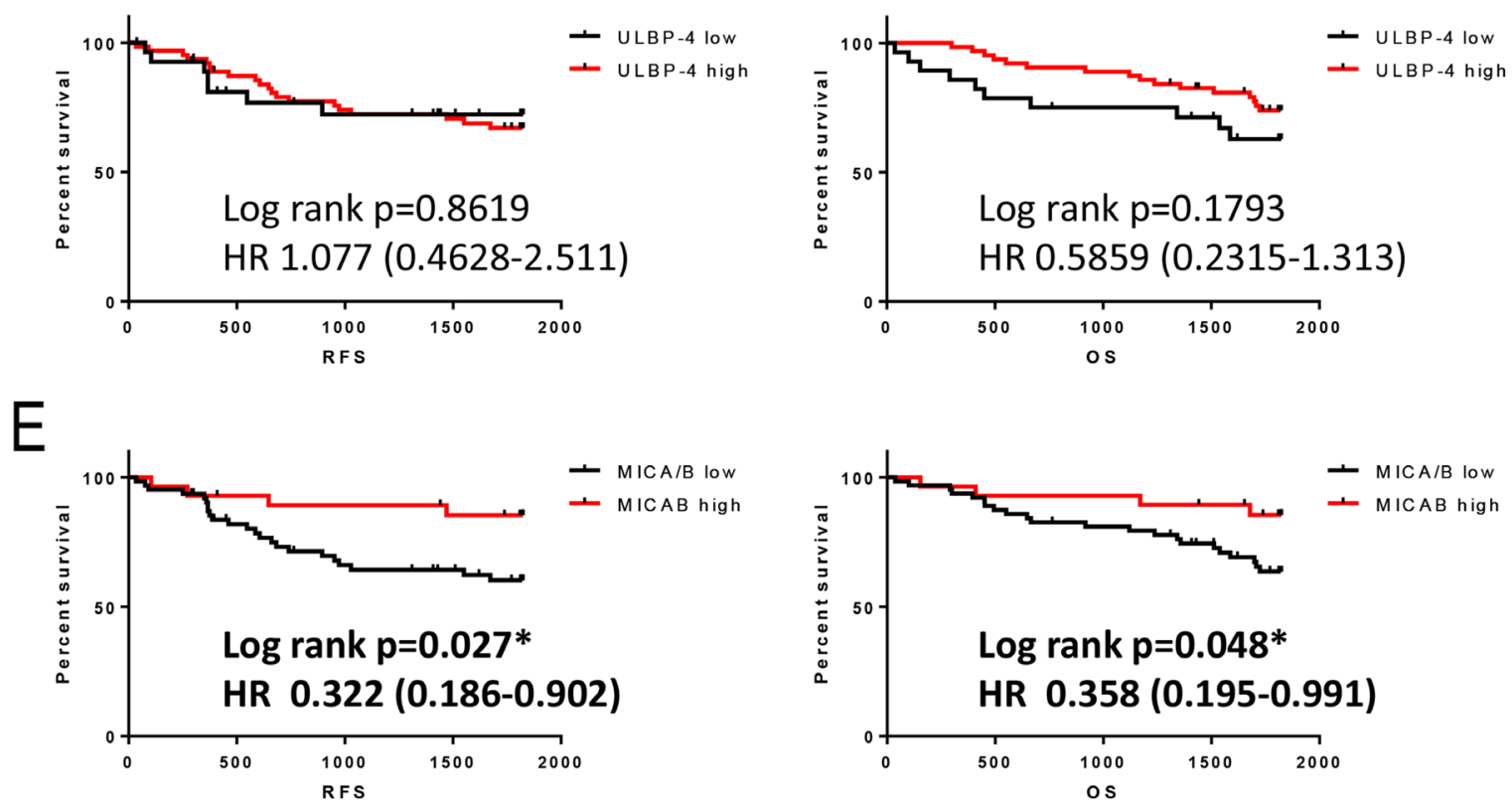

Figure 2: Association of NKG2D ligand expression in NSCLC with RFS and OS. Kaplan-Meier plots showing RFS or OS in patients with lower or higher expression of (A) ULBP1, (B) ULBP2/5/6, (C) ULBP3, and (D) ULBP4, (E) updated data for MICA/B. HR: Hazard ratio. ${ }^{*} \mathrm{p}<0.05$ 
Table 2: Cox proportional hazard model for RFS (n=91)

\begin{tabular}{lcccc}
\hline & \multicolumn{2}{c}{ Univariate } & \multicolumn{2}{c}{ Multivariate } \\
\cline { 2 - 5 } & Hazard ratio (95\% CI) & $\boldsymbol{p}$ value & Hazard ratio (95\% CI) & $\boldsymbol{p}$ value \\
\hline Gender (male vs female) & $0.787(0.353-1.753)$ & 0.558 & $0.833(0.195-3.566)$ & 0.806 \\
Smokinga (non- vs smoker) & $1.107(0.507-2.418)$ & 0.799 & $1.266(0.265-6.044)$ & 0.767 \\
Histology (Ad vs Sq) & $1.153(0.436-3.045)$ & 0.775 & $2.083(0.714-6.077)$ & 0.179 \\
Pleural invasion (neg vs pos) & $1.723(0.806-3.682)$ & 0.160 & $1.305(0.458-3.718)$ & 0.618 \\
Lymphatic invasion (neg vs pos) & $4.940(2.295-10.631)$ & $<\mathbf{0 . 0 0 1}^{*}$ & $1.787(0.533-6.000)$ & 0.347 \\
Vascular invasion (neg vs pos) & $2.721(1.643-4.506)$ & $<\mathbf{0 . 0 0 1}^{*}$ & $1.178(0517-2.685)$ & 0.697 \\
Lymphnode metastasis (neg vs pos) & $7.852(3.606-17.100)$ & $<\mathbf{0 . 0 0 1}^{*}$ & $4.779(1.487-15.361)$ & $\mathbf{0 . 0 0 9}^{*}$ \\
Category (1,2 vs 3) & $0.604(0.255-1.4312)$ & 0.252 & $0.580(0.236-1.425)$ & 0.235 \\
\hline
\end{tabular}

a:Data not available for one patient.

RFS: recurrence free survival, CI: confidence interval, Ad: adenocarcinoma, Sq: squamous cell carcinoma. *: $p>0.05$

in immune checkpoint targeted therapy remains unclear. In this study, we have shown that approximately half or more of NSCLC cases express ULBP1, ULBP-2/5/6 or ULBP4, while less than a quarter of cases express ULBP3. Interestingly, ULBP1 or ULBP2/5/6 tend to be overexpressed in lung squamous cell carcinoma, while ULBP4 is largely overexpressed in lung adenocarcinoma. We previously showed that MICA/B overexpression in NSCLC cells was independently associated with a good prognosis in terms of RFS, and the updated data showed that MICA/B overexpression is useful for the prediction of improved clinical outcomes in terms of both RFS and OS. On the other hand, overexpression of ULBP1-6 did not show any impact on either RFS or OS. Moreover, our result showed that the number of overexpressed NKG2D ligands had no impact on clinical outcomes, although several studies showed that ULBP overexpression could predict better prognosis in patients with breast cancer [12], cervical cancer [13], and hepatocellular carcinoma $[14,15]$. Following the assessment of multiple immune parameters among NKG2D ligands, cluster analysis showed that a subgroup, which was mainly characterized by either ULBP1 or ULBP $2 / 5 / 6$ high expressing but ULBP4 low expressing tumors, showed poor overall survival. Surprisingly, category 3 characteristics are a stronger prognostic factor than lymph node metastasis in terms of OS, suggesting that further study of multiple immune parameters could be a more useful classification than the TNM staging system.

The present study could also provide some ideas for the development of ULBP targeted therapies. Our data suggested that an antibody type drug targeting ULBP1 or ULBP2/5/6 might be appropriate for the treatment of squamous cell carcinoma whereas one targeting ULBP4 might be useful for treating adenocarcinoma, as more than a half of studied tumors expressed each of these molecules, respectively. Another promising strategy is NKG2D expressing Chimeric Antigen Receptor engineered (CAR) cell therapy $[21,22]$. NKG2D expressing CAR cells could recognize the tumor cells which express at least one
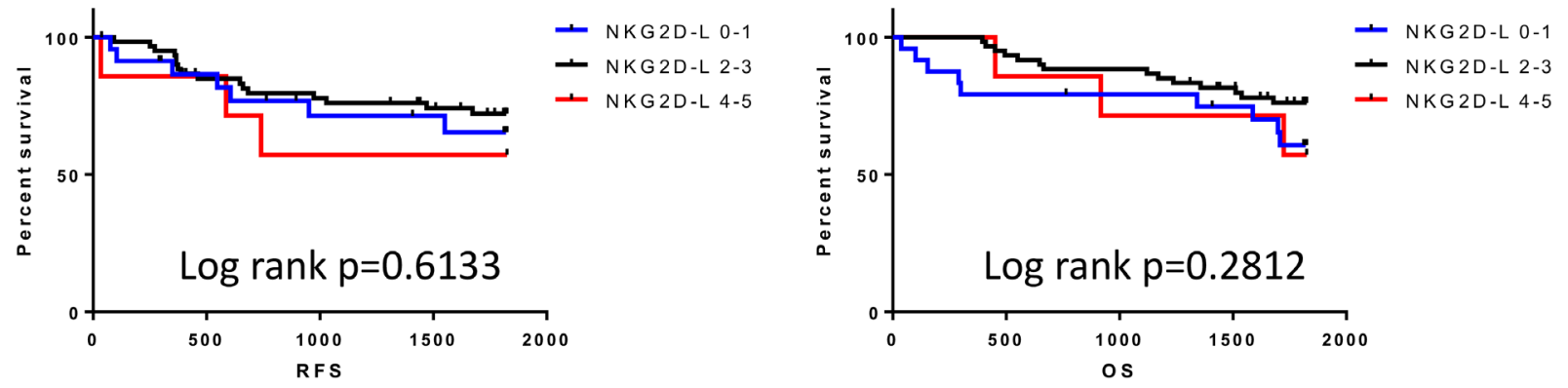

Figure 3: Survival outcomes for patients classified by the number of overexpressing NKG2D ligands. NKG2D-L 0-1: none or one NKG2D ligand expressing tumor, NKG2D-L 2-3: 2 or 3 NKG2D ligand expressing tumor, NKG2D-L 4-5: 4 or 5 NKG2D ligand expressing tumor. 

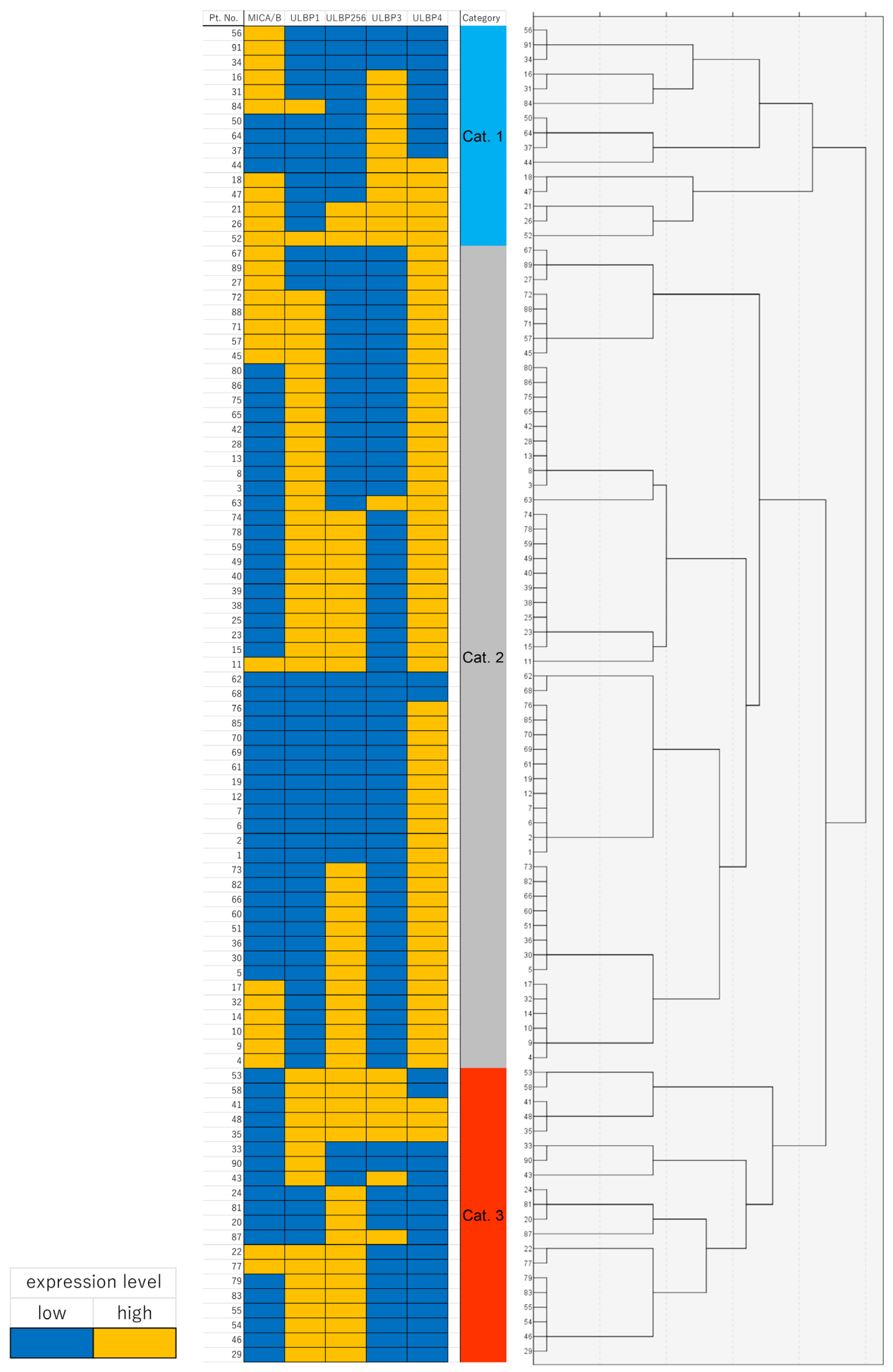

Figure 4: Heat map of immunohistochemical protein expression of MICA/B, ULBP1, ULBP2/5/6, ULBP3, and ULBP4 in the cluster map. The consensus matrix is used as the similarity matrix to define the final clusters. 
Table 3: Cox proportional hazard model for OS $(n=91)$

\begin{tabular}{|c|c|c|c|c|}
\hline & \multicolumn{2}{|l|}{ Univariate } & \multicolumn{2}{|l|}{ Multivariate } \\
\hline & Hazard ratio $(95 \% \mathrm{CI})$ & $p$ value & Hazard ratio (95\% CI) & $p$ value \\
\hline Gender (male vs female) & $0.583(0.245-1.387)$ & 0.223 & $0.778(0.162-3.734)$ & 0.753 \\
\hline Smoking ${ }^{\mathrm{a}}$ (non- vs smoker) & $1.812(0.762-4.313)$ & 0.179 & $1.516(0.300-7.661)$ & 0.615 \\
\hline Histology (Ad vs Sq) & $0.847(0.340-2.110)$ & 0.722 & $1.451(0.522-4.029)$ & 0.475 \\
\hline Pleural invasion (neg vs pos) & $2.476(1.145-5.356)$ & $0.021^{*}$ & $2.034(0.776-5.334)$ & 0.149 \\
\hline Lymphatic invasion (neg vs pos) & $3.011(1.392-6.512)$ & $0.005^{*}$ & $1.503(0.490-4.612)$ & 0.476 \\
\hline Vascular invasion (neg vs pos) & $2.180(1.305-3.644)$ & $0.003^{*}$ & $1.088(0.519-2.279)$ & 0.824 \\
\hline Lymphnode metastasis (neg vs pos) & $3.737(1.710-8.166)$ & $0.001^{*}$ & $2.643(0.919-7.603)$ & 0.071 \\
\hline Category (1,2 vs 3$)$ & $0.341(0.154-0.753)$ & $0.008^{*}$ & $0.329(0.144-0.753)$ & $0.009^{*}$ \\
\hline
\end{tabular}

a: Data not available for one patient.

OS: Overall survival, CI: confidence interval, Ad: adenocarcinoma, Sq: squamous cell carcinoma.

*: $p>0.05$

NKG2D ligand on their surface, independent of the type of NKG2D ligand. The present study found only 2 cases $(2.2 \%)$ with an all negative phenotype of NKG2D ligand expression, suggesting that over $90 \%$ of tumors might be good targets for NKG2D-CAR cell therapy.

In conclusion, we have investigated the expression pattern of ULBP1-6 in resected NSCLC. Although the
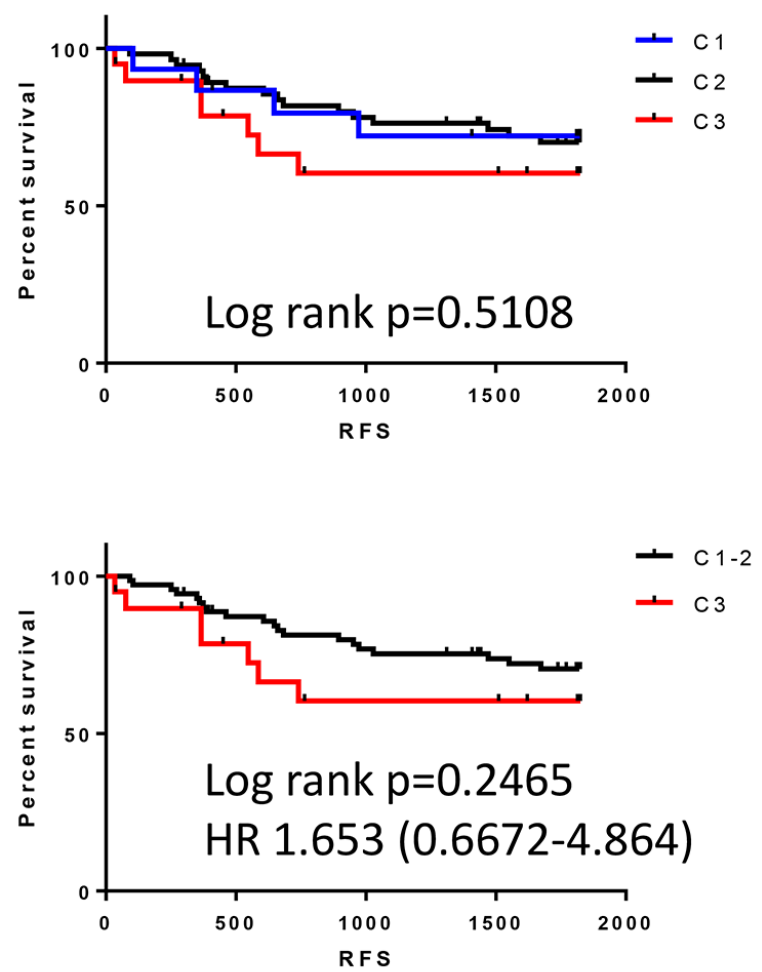

ULBP expression pattern has no impact on the prognosis of NSCLC, histological subtypes are strongly correlated with ULBP expression pattern. To develop an NKG2D ligand targeted therapy using monoclonal antibodies, patients should be selected by considering the tumor histological subtype or the expression status of the NKG2D ligand. Moreover, NKG2D expressing CAR-T
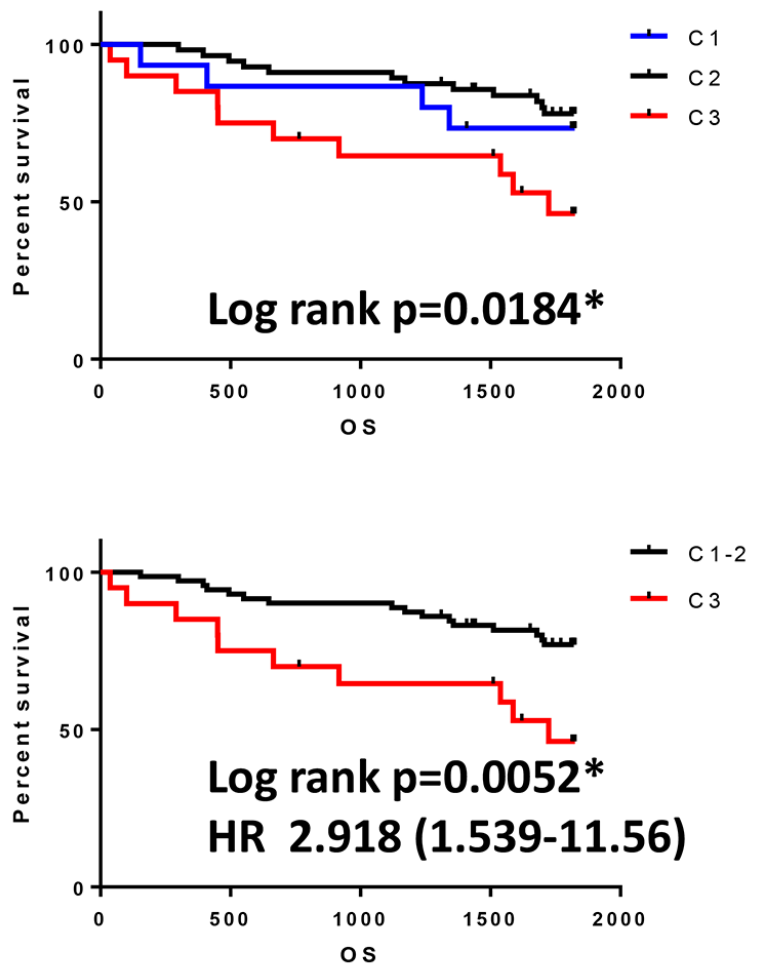

Figure 5: Survival outcomes in patients classified by cluster analysis. C1: category 1, C2: category 2, C3: category 3, C1-2: Category 1 or 2 . HR: Hazard ratio. ${ }^{*} \mathrm{p}<0.05$ 
or CAR-NK cells have good potential for the treatment of both lung adenocarcinoma and lung squamous cell carcinoma. The TNM system is well established for predicting clinical outcomes. However, cluster analysis for classification via multiple immune parameters might be a superior predictive system if the number of immune parameters is increased.

\section{MATERIALS AND METHODS}

\section{Patients and specimens}

The present study was approved by the Kawasaki Medical School ethics committee (No. 1227-4) and written informed consent was obtained from all patients before surgery for the use of resected specimens. Enrollment criteria of the patients and routine post-operative checkup details were previously described [16]. To compare the impact of MICA/B and ULBPs on the clinical outcome of patients with NSCLC, the follow-up period for MICA/B was extended from our previous study [16] to match the length of follow-up for ULBPs. Patients characteristics are shown in Supplementary Table 1.

\section{Immunohistochemical staining}

Our study included formaldehyde-fixed, paraffinembedded NSCLC specimens which were collected from pStage IA-IIIA patients. ULBP1, ULBP3, and ULBP4 expression was determined by performing immunohistochemical staining for tissue samples from NSCLC patients using a mouse monoclonal antiULBP1 antibody (clone 3F1, Santa Cruz), anti-ULBP-3 antibody (clone D-1, Santa Cruz), and an anti-ULBP-4 antibody (clone \#709116, R\&D systems), respectively, according to a previously described protocol $[16,18]$. ULBP2/5/6 expression was evaluated by performing immunohistochemical staining using a goat antiULBP2/5/6 polyclonal antibody (R\&D systems) and anti-Goat HRP-DAB Cell \& Tissue Staining Kit (R\&D systems). Mesothelioma or lung cancer tissue was used as a positive control for MICA/B, ULBP1, ULBP2/5/6, ULBP4, while bronchus was used as an internal control for ULBP3. The primary antibody was omitted from the negative control (Supplementary Table 2). Tissue slides were counterstained with hematoxylin, following which they were examined by two investigators who had no prior knowledge of the corresponding clinicopathological data. Cytosolic or membrane intensity of immunoreactivity was scored by the investigators. The intensity scoring for staining was defined as follows: "0": no staining, "1+": weak staining that was visible only with high magnification, " $2+$ ": moderate staining (between $1+$ and $3+$ ), and " $3+$ ": strong staining that was visible with low magnification. The histoscore (H-score) was calculated according to the following formula: $1 \times(\%$ cells $1+)+2 \times$
$(\%$ cells $2+)+3 \times(\%$ cells $3+)[19]$. The expression levels of ULBPs were defined as follows: "Score 0": H-score 0, "Score 1": H-score 1-99, “Score 2": H-score 100-199, "Score 3": H-score 200-300.

\section{Statistical analysis}

ROC curves for ULBPs in order to predict NSCLC recurrence were generated to determine the expected cutoff value that yielded optimal sensitivity and specificity. Chi-square tests or Fischer exact tests were performed to evaluate the relationship between ULBP expression levels and patient characteristics. Kaplan-Meier survival analysis was used to determine the association between ULBP expression and RFS or OS until death or last follow-up; the significance of the differences in RFS or OS between groups was assessed by log-rank test using GraphPad Prism 6.01 (GraphPad Software, La Jolla, CA). Cluster analysis used for the classification of our patients into subgroups based on the expression pattern of NKG2D ligands including MICA/B was performed using SPSS statistical package 17.0 (SPSS, Chicago, IL). Univariate and multivariate analyses were performed using the Cox proportional hazards model in order to identify independent prognostic factors. Statistical analyses were also performed using the SPSS statistical package 17.0. In all cases, $p<0.05$ was considered significant. The follow up period was set to a maximum of 5 years (1825 days). The median length of follow up was 1522 days (range, 37 to 1825 days) for all patients and the last follow-up date was October 6, 2017.

\section{Abbreviations}

Ad, Adenocarcinoma; CAR-T, Chimeric Antigen Receptor engineered (CAR) T cell; CAR-NK, Chimeric Antigen Receptor engineered (CAR) NK cell; CEA, Carcinoembryonic antigen; CI, Confidence interval; FDG-PET/CT, ${ }^{18} \mathrm{~F}$-fluorodeoxyglucose positron emission tomography/computed tomography; HR, Hazard ratio; MICA/B, MHC class I chain-related molecule A and B; NKG2D, NK group 2, member D; NSCLC, Non-smallcell lung cancer; OS, Overall survival; PD-1, Programmed cell death-1; PD-L1, Programmed cell death-1 ligand 1; ROC, Receiver operating characteristic; RFS, Recurrence free survival; Sq, Squamous cell carcinoma; SUVmax, Maximum standard uptake value; ULBP, UL16-binding protein;

\section{Author contributions}

Conceived and designed the experiments: R Okita Performed the experiments: R Okita. Analyzed the data: R Okita and A Maeda. Collected clinical data and samples: R Okita, Y Nojima, A Maeda, S Saisho, K Shimizu, and M Nakata. 
Contributed reagents/materials/analysis tools: $\mathrm{R}$ Okita and M Nakata.

Wrote the manuscript: R Okita, and M Nakata.

All authors read and approved the final manuscript.

\section{ACKNOWLEDGMENTS}

We thank the staff of the Tissue Culture \& Immunology and the Tissue Biology \& Electron Microscopy Research Centers (Kawasaki Medical School) for providing technical assistance. The authors thank Editage for the language editing.

\section{CONFLICTS OF INTEREST}

Dr. M. Nakata received research funding from Kyowa Kirin, Taiho Pharma, Ono Pharma, and Nihon Medi-Physics for research outside the scope of the submitted work. All other authors declare no conflicts of interest.

\section{FUNDING}

This work was supported by the Japan Society for the Promotion of Science (JSPS) Kakenhi Grant (25462189 and 16K10696) to Riki Okita.

\section{Compliance with Ethical Standards}

Our research was approved by the Kawasaki Medical School ethics committee (No. 1227-4).

\section{REFERENCES}

1. Jemal A, Siegel R, Ward E, Hao Y, Xu J, Thun MJ. Cancer statistics, 2009. CA Cancer J Clin. 2009; 59: 225-49. https://doi.org/10.3322/caac.20006. [PubMed]

2. Goldstraw P, Crowley J, Chansky K, Giroux DJ, Groome PA, Rami-Porta R, Postmus PE, Rusch V, Sobin L, and International Association for the Study of Lung Cancer International Staging Committee, and Participating Institutions. The IASLC Lung Cancer Staging Project: proposals for the revision of the TNM stage groupings in the forthcoming (seventh) edition of the TNM Classification of malignant tumours. J Thorac Oncol. 2007; 2: 706-14. https://doi.org/10.1097/JTO.0b013e31812f3c1a. [PubMed]

3. Al-Shibli KI, Donnem T, Al-Saad S, Persson M, Bremnes RM, Busund LT. Prognostic effect of epithelial and stromal lymphocyte infiltration in non-small cell lung cancer. Clin Cancer Res. 2008; 14: 5220-7. https://doi.org/10.1158/1078-0432.CCR-08-0133. [PubMed]

4. So T, Takenoyama M, Sugaya M, Yasuda M, Eifuku R, Yoshimatsu T, Osaki T, Yasumoto K. Unfavorable prognosis of patients with non-small cell lung carcinoma associated with HLA-A2. Lung Cancer. 2001; 32: 39-46. https://doi.org/10.1016/S0169-5002(00)00204-X. [PubMed]

5. Brahmer J, Reckamp KL, Baas P, Crino L, Eberhardt WE, Poddubskaya E, Antonia S, Pluzanski A, Vokes EE, Holgado E, Waterhouse D, Ready N, Gainor J, et al. Nivolumab versus docetaxel in advanced squamous-cell non-small-cell lung cancer. N Engl J Med. 2015; 373: 123 35. https://doi.org/10.1056/NEJMoa1504627. [PubMed]

6. Borghaei H, Paz-Ares L, Horn L, Spigel DR, Steins M, Ready NE, Chow LQ, Vokes EE, Felip E, Holgado E, Barlesi F, Kohlhaufl M, Arrieta O, et al. Nivolumab versus docetaxel in advanced nonsquamous non-smallcell lung cancer. N Engl J Med. 2015; 373: 1627-39. https://doi.org/10.1056/NEJMoa1507643. [PubMed]

7. Mazzaschi G, Madeddu D, Falco A, Bocchialini G, Goldoni M, Sogni F, Armani G, Lagrasta CA, Lorusso B, Mangiaracina C, Vilella R, Frati C, Alfieri R, et al. Low PD-1 expression in cytotoxic $\mathrm{CD} 8(+)$ tumorinfiltrating lymphocytes confers an immune-privileged tissue microenvironment in NSCLC with a prognostic and predictive value. Clin Cancer Res. 2018; 24: 407-19. https://doi.org/10.1158/1078-0432.CCR-17-2156. [PubMed]

8. Subrahmanyam PB, Dong Z, Gusenleitner D, GiobbieHurder A, Severgnini M, Zhou J, Manos M, Eastman LM, Maecker HT, Hodi FS. Distinct predictive biomarker candidates for response to anti-CTLA-4 and anti-PD-1 immunotherapy in melanoma patients. J Immunother Cancer. 2018; 6: 18. https://doi.org/10.1186/s40425-0180328-8. [PubMed]

9. Lanier LL. A renaissance for the tumor immunosurveillance hypothesis. Nat Med. 2001; 7: 1178-80. https://doi.org/10.1038/nm1101-1178. [PubMed]

10. Bauer S, Groh V, Wu J, Steinle A, Phillips JH, Lanier LL, Spies T. Activation of NK cells and T cells by NKG2D, a receptor for stress-inducible MICA. Science. 1999; 285: 727-9. https://doi.org/10.1126/science.285.5428.727. [PubMed]

11. Rimawi MF, Aleixo SB, Rozas AA, Nunes de Matos Neto J, Caleffi M, Figueira AC, Souza SC, Reiriz AB, Gutierrez C, Arantes H, Uttenreuther-Fischer MM, Solca F, Osborne CK. A Neoadjuvant, Randomized, openlabel phase II trial of afatinib versus trastuzumab versus lapatinib in patients with locally advanced HER2-positive breast cancer. Clin Breast Cancer. 2015; 15: 101-9. https://doi.org/10.1016/j.clbc.2014.11.004. [PubMed]

12. de Kruijf EM, Sajet A, van Nes JG, Putter H, Smit VT, Eagle RA, Jafferji I, Trowsdale J, Liefers GJ, van de Velde CJ, Kuppen PJ. NKG2D ligand tumor expression and association with clinical outcome in early breast cancer patients: an observational study. BMC Cancer. 2012; 12: 24. https://doi.org/10.1186/1471-2407-12-24. [PubMed]

13. Cho H, Chung JY, Kim S, Braunschweig T, Kang TH, Kim J, Chung EJ, Hewitt SM, Kim JH. MICA/B and ULBP1 
NKG2D ligands are independent predictors of good prognosis in cervical cancer. BMC Cancer. 2014; 14: 957. https://doi.org/10.1186/1471-2407-14-957. [PubMed]

14. Kamimura H, Yamagiwa $S$, Tsuchiya A, Takamura M, Matsuda Y, Ohkoshi S, Inoue M, Wakai T, Shirai Y, Nomoto M, Aoyagi Y. Reduced NKG2D ligand expression in hepatocellular carcinoma correlates with early recurrence. J Hepatol. 2012; 56: 381-8. https://doi.org/10.1016/j.jhep.2011.06.017. [PubMed]

15. Fang L, Gong J, Wang Y, Liu R, Li Z, Wang Z, Zhang Y, Zhang C, Song C, Yang A, Ting JP, Jin B, Chen L. MICA/B expression is inhibited by unfolded protein response and associated with poor prognosis in human hepatocellular carcinoma. J Exp Clin Cancer Res. 2014; 33: 76. https://doi.org/10.1186/s13046-014-0076-7. [PubMed]

16. Okita R, Yukawa T, Nojima Y, Maeda A, Saisho S, Shimizu K, Nakata M. MHC class I chain-related molecule A and $\mathrm{B}$ expression is upregulated by cisplatin and associated with good prognosis in patients with non-small cell lung cancer. Cancer Immunol Immunother. 2016; 65: 499-509. https://doi.org/10.1007/s00262-016-1814-9. [PubMed]

17. Yamaguchi K, Chikumi H, Shimizu A, Takata M, Kinoshita N, Hashimoto K, Nakamoto M, Matsunaga S, Kurai J, Miyake N, Matsumoto S, Watanabe M, Yamasaki A, et al. Diagnostic and prognostic impact of serum-soluble UL16-binding protein 2 in lung cancer patients. Cancer Sci. 2012; 103: 1405-13. https://doi.org/10.1111/j.1349-7006.2012.02330.x. [PubMed]

18. Okita R, Maeda A, Shimizu K, Nojima Y, Saisho S, Nakata M. PD-L1 overexpression is partially regulated by EGFR/HER2 signaling and associated with poor prognosis in patients with non-small-cell lung cancer. Cancer Immunol Immunother. 2017; 66: 865-76. https://doi.org/10.1007/s00262-017-1986-y. [PubMed]

19. Nunez MI, Behrens C, Woods DM, Lin H, Suraokar M, Kadara H, Hofstetter W, Kalhor N, Lee JJ, Franklin W, Stewart DJ, Wistuba II. High expression of folate receptor alpha in lung cancer correlates with adenocarcinoma histology and EGFR [corrected] mutation. J Thorac Oncol. 2012; 7: 833-40. https://doi.org/10.1097/JTO.0b013e31824de09c. [PubMed]

20. Kurose K, Ohue Y, Wada H, Iida S, Ishida T, Kojima T, Doi T, Suzuki S, Isobe M, Funakoshi T, Kakimi K, Nishikawa $\mathrm{H}$, Udono H, et al. Phase Ia study of FoxP3+ CD4 Treg depletion by infusion of a humanized anti-CCR4 antibody, KW-0761, in cancer patients. Clin Cancer Res. 2015; 21: 4327-36. https://doi.org/10.1158/1078-0432.CCR-15-0357. [PubMed]

21. Lehner M, Gotz G, Proff J, Schaft N, Dorrie J, Full F, Ensser A, Muller YA, Cerwenka A, Abken H, Parolini $\mathrm{O}$, Ambros PF, Kovar $\mathrm{H}$, et al. Redirecting $\mathrm{T}$ cells to Ewing's sarcoma family of tumors by a chimeric NKG2D receptor expressed by lentiviral transduction or mRNA transfection. PLoS One. 2012; 7: e31210. https://doi.org/10.1371/journal.pone.0031210. [PubMed]

22. Chang YH, Connolly J, Shimasaki N, Mimura K, Kono $\mathrm{K}$, Campana D. A chimeric receptor with NKG2D specificity enhances natural killer cell activation and killing of tumor cells. Cancer Res. 2013; 73: 1777-86. https://doi.org/10.1158/0008-5472.CAN-12-3558.

[PubMed] 